\title{
From synchrotrons for XFELs: the soft x-ray near-edge spectrum of the ESCA molecule
}

\author{
S.L. Sorensen ${ }^{1}$, X. Zheng ${ }^{2}$, S.H. Southworth ${ }^{3}$, M. Patanen ${ }^{4}$, \\ E. Kokkonen ${ }^{4,5}$, B. Oostenrijk ${ }^{1}$, O. Travnikova ${ }^{6}, \mathbf{T}$. \\ Marchenko $^{6}$, M. Simon ${ }^{6}$, C. Bostedt ${ }^{3,7,8}$, G. Doumy ${ }^{3}$, L. \\ Cheng $^{2}$, L. Young ${ }^{3,9}$ \\ ${ }^{1}$ Department of Physics, Lund University, Box 118, 22100 Lund, Sweden \\ ${ }^{2}$ Department of Chemistry, Johns Hopkins University, Baltimore USA \\ ${ }^{3}$ Chemical Sciences and Engineering Division, Argonne National Laboratory, \\ 9700 S Cass Avenue, Lemont, IL 60439, United States of America \\ ${ }^{4}$ Faculty of Science, Nano and Molecular Systems Research Unit, University of \\ Oulu, Box 3000, FIN-90014 Oulu, Finland \\ ${ }^{5}$ MAX IV Laboratory, Lund University, Box 118, SE-221 00 Lund, Sweden \\ ${ }^{6}$ Sorbonne Université, CNRS, Laboratoire de Chimie Physique - Matière et \\ Rayonnement, LCPMR, F-75005 Paris, France \\ ${ }^{7}$ Paul Scherrer Institut, Switzerland \\ ${ }^{8}$ LUXS Laboratory for Ultrafast X-ray Sciences, École Polytechnique Fédérale \\ de Lausanne, Switzerland \\ ${ }^{9}$ Department of Physics and James Franck Institute, The University of Chicago, \\ Chicago, 60637, USA \\ E-mail: young@anl.gov
}

\begin{abstract}
A predictive understanding of soft x-ray near-edge absorption spectra of small molecules is an enduring theoretical challenge and of current interest for x-ray probes of molecular dynamics. We report the experimental absorption spectrum for the ESCA molecule (ethyl trifluoroacetate) near the carbon 1s absorption edge between 285-300 eV. The ESCA molecule with four chemically distinct carbon sites has previously served as a theoretical benchmark for photoelectron spectra and now for photoabsorption spectra. We report a simple edge-specific approach for systematically expanding standard basis sets to properly describe diffuse Rydberg orbitals and the importance of triple excitations in equation-of-motion coupled-cluster calculations of the energy interval between valence and Rydberg excitations.
\end{abstract}

\section{Introduction}

X-ray free-electron lasers (XFELs) $[1,2,3,4,5,6]$ and table-top sources of x-rays based upon high harmonic generation (HHG) $[7,8]$ have over the past decade energized the field of ultrafast atomic and molecular dynamics [9]. For XFELs, the intense tunable x-ray pulses that span wavelengths from $\sim 40$ to $0.5 \AA$ with sub-femtosecond pulse durations $[10,11,12]$ and multi-pulse, multi-color options $[13,14,15]$ allow one to investigate electronic and nuclear dynamics on their natural timescales. Using pump-probe schemes, one can not only study valence-excited dynamics, e.g. the mechanism of photoprotection of DNA bases via intersystem crossing [16], but also 
inner-shell dynamics where competition between localized Auger decay and chargetransfer mediates dissociation in small molecules [17].

With the enormous versatility of pump and probe x-ray pulses, it is natural to contemplate the optimal observable for nuclear and electronic orbital dynamics [18] and even consider nonlinear multi-dimensional spectroscopy techniques [19, 20]. Two straightforward single-photon methods, photoelectron spectroscopy and photoabsorption spectroscopy are universally applicable. X-ray photoelectron spectroscopy (XPS) will yield binding energies of core and valence orbitals and has been widely used for chemical analysis, as exemplified by the iconic studies by Siegbahn $[21,22]$. This method, Electron Spectroscopy for Chemical Analysis (ESCA), was showcased by the XPS spectrum of ethyl trifluoroacetate, now commonly known as the ESCA molecule, where the dramatic shifts of the $\mathrm{C} 1 s$ binding energies illustrate the connection between the local chemical environment and the binding energy. The site-specificity of the four non-identical carbons is encoded in their binding energies which differ by $\sim 8 \mathrm{eV}$ from the electronegative $\mathrm{CF}_{3}$ end to the $\mathrm{CH}_{3}$ end, as shown in Fig. 1. A more recent combined experimental and theoretical study shows that the high-resolution $\mathrm{C} 1 s$ photoelectron spectrum also possesses some limited sensitivity to the conformational state and nuclear dynamics associated with photoionization [23]. However, it was the intrinsic site-specific nature of the binding energies that motivated our search for chemical site-specificity in bond-breakage in the ESCA molecule using the photoelectron-photoion-photoion coincidence method [24]. We found limited site specificity. For all four carbon ionization sites, Auger decay weakens the same bonds and transfers the two charges to opposite ends of the molecule, which leads to a rapid dissociation into three fragments, followed by further fragmentation steps.

So the general question naturally arises, what is the mechanism by which an initially localized $1 s$ hole delocalizes across the molecule? Can we probe the evolution of the valence hole density across the molecule via snapshots of its near-edge x-ray absorption fine structure (NEXAFS) spectrum? Of course, NEXAFS has long been a powerful technique for chemical analysis [25] and has been more recently applied for time-resolved studies of evolving valence electronic structure using both HHG [26, 27] and XFEL sources $[16,28]$. Somewhat surprisingly, to date there is no published softx-ray absorption spectrum for the iconic ESCA molecule, ethyl trifluoroacetate. As our results show, the photoabsorption spectrum near the carbon K-edge is much more complicated than its photoelectron spectrum - with no one-to-one correspondence between the carbon site and the absorption spectrum (see Fig. 1). The ability to theoretically assign complex NEXAFS spectra, obtained reliably from synchrotron studies, is of utmost importance for current studies of electronic and structural dynamics with XFELs $[28,29,30]$. Here we systematically tackle this challenge for the ESCA molecule.

Despite the complexity associated with near-edge absorption spectroscopy, there has been considerable progress in theoretical descriptions driven largely by advances in light source technology [31, 32]. Of particular relevance to the present work is the application of equation-of-motion coupled-cluster (EOM-CC) techniques [33, 34], which can be systematically converged towards the right answer, to calculations of core-level spectroscopy. The earlier work [35, 36, 37, 38, 39] shows promise of the EOM-CC methods for obtaining accurate core excitation energies, but was hampered by the difficulty of converging EOM-CC equations for core-excited states. With an elegant approach of introducing the core-valence separation (CVS) scheme [40] into the EOM-CC formulation [41], the CVS-EOM-CC methods have recently evolved into 
powerful tools for calculations of core ionization and excitation energies, photoelectron spectra, NEXAFS spectra, and resonant inelastic x-ray scattering (RIXS) spectra $[42,43,44,45,46,47,48,49,50,51,52]$. A unique appealing feature of CVS-EOM$\mathrm{CC}$ is the capability of providing systematically improved results using the hierarchy of EOM-CC methods. Namely, while the popular EOM-CC singles and doubles (EOMCCSD) method is capable of providing useful results $[53,46,54,55,56]$, the inclusion of higher excitations in the CC hierarchy further enables systematic improvement of computational results, which has the desired potential of paving the way to calculations that are essentially quantitative $[45,52,48,57,58]$. This has been exploited in the present study to assess the reliability of computational simulation for the fairly complicated NEXAFS spectrum of the ESCA molecule.

In this paper we report the experimental near-edge absorption spectrum of ethyl trifluoroacetate (the "ESCA" molecule) and theoretically describe the origin of its features using CVS-EOM-CC methods. Our theoretical developments include 1) a simple edge-specific approach for systematically converging basis set effects to properly describe Rydberg orbitals, and, 2) the demonstration of importance of including triple excitations to obtain accurate valence-to-Rydberg energy spacings in carbonyl containing compounds. These developments, which appear to be generally applicable, are benchmarked with calculations of simple small molecules, $\mathrm{NH}_{3}, \mathrm{CO}_{2}$, and $\mathrm{CH}_{2} \mathrm{O}$, and then used for the calculations of the NEXAFS spectrum for the ESCA molecule to facilitate the assignment of the experimental spectrum.

\section{Experimental considerations}

Synchrotron measurements of absorption spectra of atoms and molecules have long contributed to the advances in theoretical methods to describe photoabsorption to bound and continuum states [59,60,61]. Relative to FELs the photon-molecule interactions are simpler, being constrained to single-photon interactions. The probability of photon absorption by an isolated single molecule is given by the product of the pulse fluence and its absorption cross section. For monochromatic synchrotron radiation, with a typical $10^{6}$ photons per pulse focused to $1 \mu \mathrm{m}^{2}$ and a cross section of $1 \mathrm{Mb}$, the probability of one photon being absorbed within a photon pulse is $10^{-4}$ and the probability for two-photon absorption $10^{-8}$. The situation is vastly different at XFELs where one routinely obtains $10^{12}$ photons per pulse to easily saturate single-photon absorption and create multiphoton absorption [62, 63]. Detection of the single-photon x-ray absorption spectra in the gas phase at synchrotrons can be done either directly in transmission or by detection of secondary emitted particles, i.e. photons, electrons or ions. For the non-transmission measurements of photoabsorption, the observed spectral features are affected by molecular dynamics that control the branching ratios to the secondary products $[25,64]$. The spectra reported here have been obtained via total-ion-yield spectroscopy which is closely related to photoabsorption [64].

The bottom panel of Fig. 1 shows the total-ion-yield spectrum of ethyl trifluoroacetate measured on the PLEIADES beam line at the SOLEIL synchrotron radiation facility. Ions were collected in a time-of-flight spectrometer with an extraction field of about $130 \mathrm{~V} / \mathrm{cm}(200 \mathrm{~V}$ between the repeller and extractor plates, which are separated by $15.5 \mathrm{~mm})[65,66]$. The photon-energy resolution in the measurement was $25 \mathrm{meV}$, considerably less than the lifetime width of a carbon $1 \mathrm{~s}$ hole $\sim 100 \mathrm{meV}$ [67]. The photon energy was simultaneously calibrated using carbon 
From synchrotrons for XFELs: the soft x-ray near-edge spectrum of the ESCA molecule4

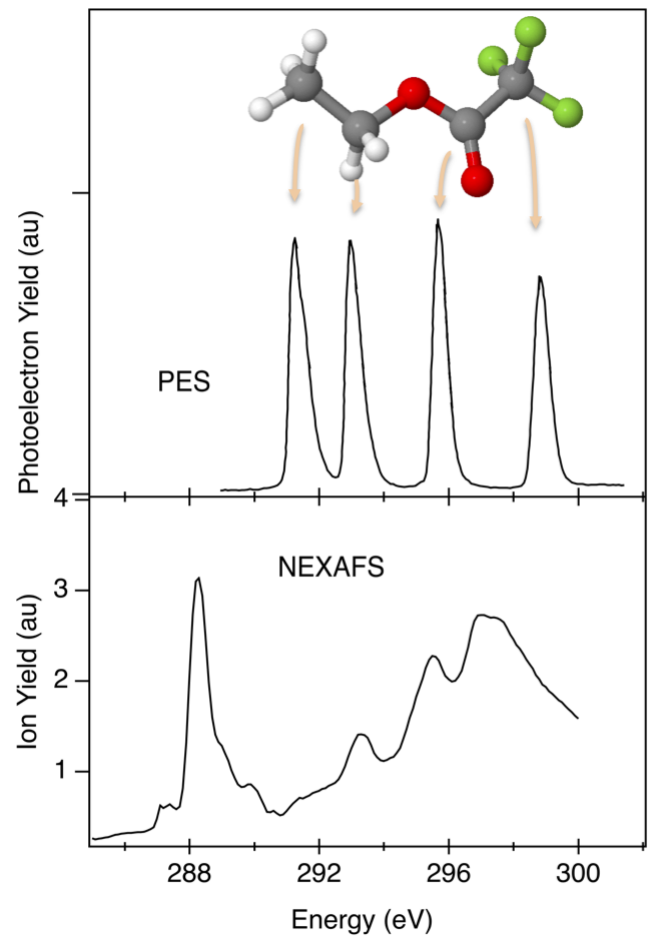

Figure 1: Comparison of photoelectron and photoabsorption spectra for the ESCA molecule. Top panel: the $\mathrm{C} 1 \mathrm{~s}$ binding energy of ethyl trifluoroacetate recorded at a photon energy of $340 \mathrm{eV}$, extracted from [23]. There is a one-to-one correspondence between the photoelectron peaks and the four distinct carbon atoms (grey), which are bonded to hydrogen (white), oxygen (red) and fluorine (green).

The $1 s$ binding energy of $\mathrm{C}_{\mathrm{CH}_{3}}$ is $291.47 \mathrm{eV}$. Bottom panel: photoabsorption via total ion yield of ethyl trifluoroacetate obtained by scanning the photon energy from 285 to $300 \mathrm{eV}$ (This work. See text for details).

dioxide in a separate ionization chamber and calibrated to the study by Adachi [68] with a precision of $\sim 50 \mathrm{meV}$. The measurement was carried out using an effusive gas jet resulting in a background pressure of $5 \times 10^{-7}$ mbar.

The $\mathrm{C}$ 1s total-ion-yield spectrum exhibits features corresponding to core electron excitation from each of the four chemically-shifted carbon sites. No previous absorption spectra have been reported in the region near the $\mathrm{C}$ 1s ionization thresholds. Several narrow absorption features are seen below the lowest carbon 1s ionization threshold, but the identification of these features and the character of the occupied valence orbitals, requires the detailed analysis described in Section 3.

\section{Computational methods}

The computational simulation of the NEXAFS spectra presented here has used corevalence separated equation-of-motion coupled-cluster (CVS-EOM-CC) [41] calculations for core excited states. The computational approaches are discussed in detail 
here and their accuracy demonstrated for $\mathrm{C}, \mathrm{N}$, and $\mathrm{O}$ edges using ammonia $\left(\mathrm{NH}_{3}\right)$ and formaldehyde $\left(\mathrm{CH}_{2} \mathrm{O}\right)$ as two benchmark molecules. Specifically, in subsection 3.1, using $\mathrm{NH}_{3}$ as a model system, we demonstrate the evolution of NEXAFS spectrum as a function of the basis set using an edge-specific approach to saturate basis set effects. Contributions from triple excitations are important for accurate calculations of NEXAFS for molecules containing a carbonyl group. Their effects are systematically studied for $\mathrm{CH}_{2} \mathrm{O}$ at both $\mathrm{C}$ 1s and $\mathrm{O}$ 1s edges, and taken into account in the calculations of ESCA molecule, as detailed in subsection 3.2. We have correlated the targeted core orbital and have kept the other core orbitals frozen in ground-state CC calculations. This is also intrinsically an edge-specific scheme, consistent with the present edge-specific approach for treating basis-set effects on Rydberg states. It has been reported that the frozen core version of CVS-EOM-CCSD (fc-CVS-EOM-CCSD) method [44] often yield accurate core excitation energies, benefiting from cancellation between errors of the frozen-core approximation and the neglect of triple excitations. However, since it is essential to include triple excitations to obtain accurate results for the ESCA molecule, it is necessary to correlate the 1s electrons of the targeted carbon in ground-state $\mathrm{CC}$ calculations. In all calculations presented here, scalar-relativistic effects have been taken into account using the spin-free exact two-component theory in its one-electron variant (SFX2C-1e) $[69,70]$ and the correlation-consistent basis sets [71] with SFX2C-1e recontraction. All calculations have been carried out using the CFOUR program package $[72,73,33,74,75,76]$. The calculations presented here for the ESCA molecule have used the structure of the $\mathrm{C}_{s}$ conformer computed in Ref. [77]. We mention that similar results have been obtained for the $\mathrm{C}_{1}$ conformer with details documented in Table 4 of the supplementary material.

EOM-CCSD density difference natural orbitals (DDNOs) have been used to enable intuitive understanding of the excitations in these core excited states. DDNOs are obtained by diagonalizing the one-electron density difference matrix, i.e., the difference between the excited state and ground state EOM-CCSD one-electron density matrices. For each core-excited state obtained in the present work, one obtains a set of DDNOs with one of them having an occupation number close to -1 (the 1s orbital excited from) and one having an occupation number close to 1 (the natural virtual orbital excited to). The latter has been plotted for twelve core excited states with the highest intensities in the NEXAFS spectrum of the ESCA molecule. For a first study using EOM-CCSD DDNOs to facilitate the analysis of NEXAFS spectra, we refer the reader to Ref. [43]. We also refer the reader to Ref. [78] as a recent perspective review on related concepts about transition natural orbitals for further discussions of orbital analysis in x-ray spectroscopy as well as Ref. [79] for DDNOs in other context.

\subsection{An edge-specific approach for treating basis set effects on core-excited Rydberg states}

Core-excited Rydberg states are inadequately accounted for in calculations without using sufficient diffuse basis functions to capture the diffuse character of these states [46]. As shown in Fig. 2 for ammonia, the use of the standard cc-pVTZ basis [71] overestimates excitation energies for the $1 s \rightarrow 3 p$ states and misses all other Rydberg states. To solve this problem, we augment the standard correlation-consistent basis sets for the targeted atom systematically with diffuse s-, p-, and d-type functions. In the following we use " +xspd" to refer to the addition of $\mathrm{x}$ sets of s-, p-, and d-type diffuse functions. 
The exponents of diffuse functions are obtained by multiplying those of the most diffuse functions in the set by a geometric factor of $1 / 3$. Interestingly, a convergence pattern has been observed. The addition of two sets of diffuse functions is required to saturate the description for the first set of Rydberg states, while each additional set of diffuse functions then serve to converge another set of Rydberg states. Namely, as demonstrated in Fig. 2, cc-pVTZ+2spd, cc-pVTZ+3spd, and cc-pVTZ+4spd sets provide converged results for $1 s \rightarrow 3 p, 1 s \rightarrow 4 p$, and $1 s \rightarrow 5 p$ transitions, respectively. Therefore, in calculations of the ESCA molecule, the cc-pVTZ+4spd set has been adopted for the targeted carbon atom together with cc-pVTZ basis sets for all other atoms to ensure an accurate description of valence transitions and the first three sets of core excited Rydberg states.

The present scheme is edge specific in that a separate EOM-CC calculation is carried out for core excited states of each atom with additional diffuse functions located on this atom, e.g., in the case of the carbon NEXAFS of the ESCA molecule, each of the four carbon edges requires one separate EOM-CC calculation. It appears to be a useful alternative to the use of molecular Rydberg functions in Refs. [46]. The latter can be combined with the frozen core version of CVS-EOM-CC methods [44] to enable calculations of core excited states of all edges with only a single groundstate CC calculation, being an edge-universal approach. On the other hand, for larger molecules, the present edge-specific approach for adding diffuse functions appears to be physically better motivated than using the center of charge as the center for molecular Rydberg functions, as the localized core hole serves as a well-defined center for core-excited Rydberg states.

\subsection{Importance and treatment of triples contributions}

The CVS-EOM-CC core ionization and excitation energies can be improved in a systematic way by increasing the rank of excitation operators [45]. The CVS-EOM coupled-cluster singles and doubles (CCSD) method has been shown to provide useful results for NEXAFS spectra $[46,54,55]$. Although EOM-CCSD calculations typically overestimate core ionization and excitation energies by $0.5-3 \mathrm{eV}$, the errors are often partially offset by the neglect of scalar-relativistic effects and correlation contributions of core electrons. Further, these errors tend not to contribute to relative shifts and, thus, do not affect the overall shape of the simulated spectra. For example, as already shown in Fig. 2, EOM-CCSD calculations describe the NEXAFS of ammonia quite well. However, a notable exception is that CVS-EOM-CCSD calculations have been reported to significantly overestimate the separation between the carbon (or oxygen) $1 s \rightarrow \pi^{*}$ transition and the corresponding Rydberg transitions in the formaldehyde $\left(\mathrm{CH}_{2} \mathrm{O}\right)$ molecule [46]. Here we show that the inclusion of triple excitations enables accurate calculations of these relative shifts. The carbon and oxygen 1s core excitation energies of $\mathrm{CH}_{2} \mathrm{O}$ obtained from CVS-EOM-CCSD [33] and CC single doubles and triples (CCSDT) [81, 82] calculations are summarized in Table 1. Triples corrections (the difference between CCSDT and CCSD results) to the relative shifts between $1 s \rightarrow \pi^{*}$ and the first Rydberg transitions amount to $0.44 \mathrm{eV}$ for the carbon edge and as large as $0.84 \mathrm{eV}$ for the oxygen edge. The inclusion of triples corrections reduces

the separation between the $1 s \rightarrow \pi^{*}$ transition and the first Rydberg transition from $4.68 \mathrm{eV}$ to $4.24 \mathrm{eV}$ for the carbon edge and from $5.28 \mathrm{eV}$ to $4.44 \mathrm{eV}$ for the oxy- 
From synchrotrons for XFELs: the soft x-ray near-edge spectrum of the ESCA molecule7
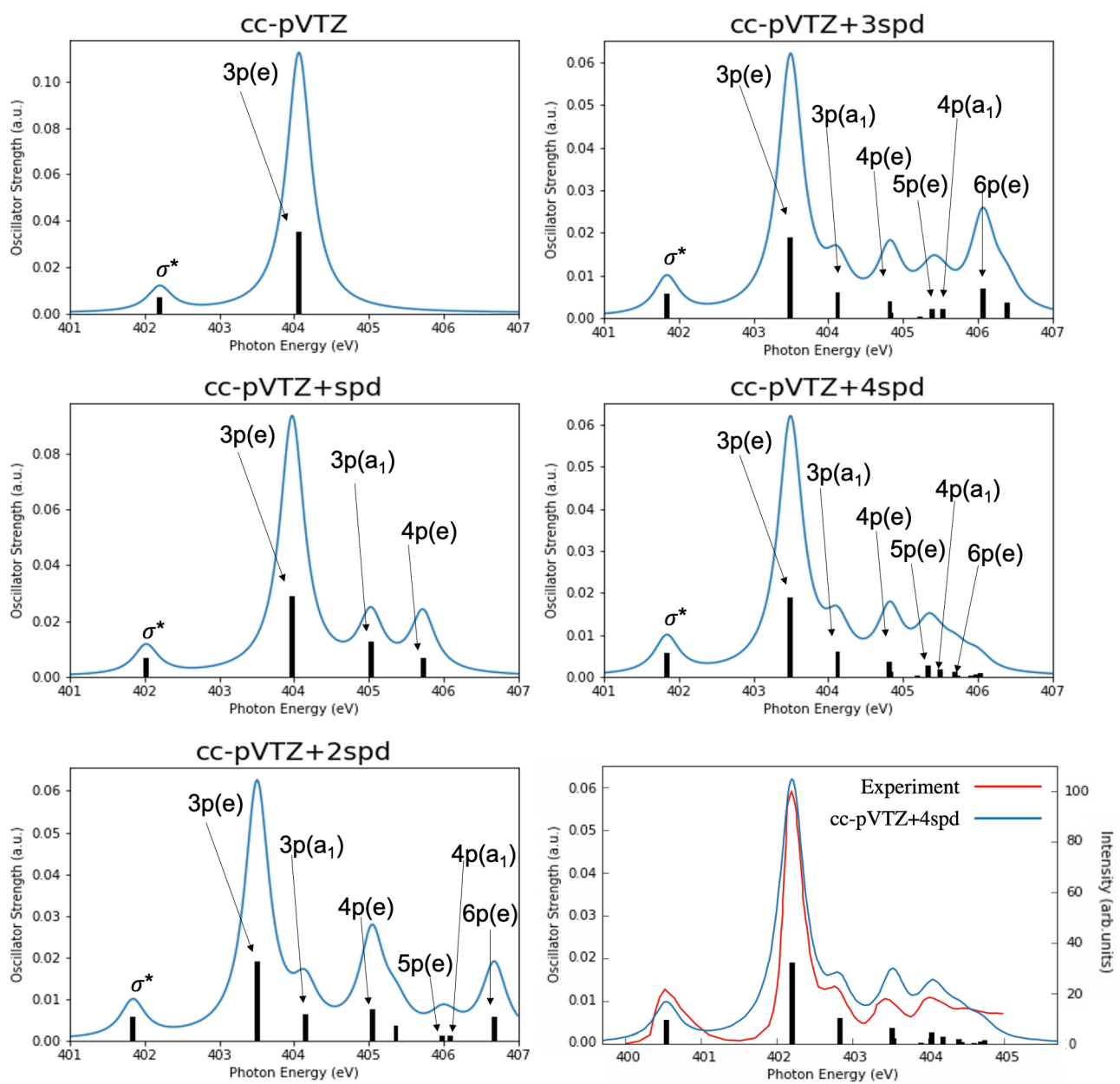

Figure 2: EOM-CCSD NEXAFS spectra for the nitrogen edge in ammonia obtained by convolution of the computed energies and oscillator strengths with a Lorentzian function with a FWHM value of $0.4 \mathrm{eV}$. Scalar-relativistic effects were taken into account using the spin-free exact two-component theory in its one-electron variant. "cc-pVTZ+xspd" $(\mathrm{x}=1,2,3,4)$ refers to augmentation of nitrogen cc-pVTZ basis sets with $\mathrm{x}$ sets of additional diffuse s-, p-, and d-type functions. In the last figure, the red solid line represents experimental spectrum [80] and the computed spectrum has been red-shifted by $1.30 \mathrm{eV}$ to align the computed $1 \mathrm{~s} \rightarrow \sigma^{*}$ transition with the experiment.

gen edge. These are to be compared with the experiment values of $4.15 \mathrm{eV}$ and 4.66 $\mathrm{eV}$. As the ESCA molecule also contains a carbonyl group, it is therefore necessary to include triples corrections to obtain accurate results for its NEXAFS spectrum. The computational cost of CCSDT scales as the eighth power of the system size, and CVS-EOM-CCSDT calculations of the ESCA molecule are beyond our current computational resources. Therefore, we have adopted the $\operatorname{EOM}-\operatorname{CCSD}(\mathrm{T})(\mathrm{a})^{*}$ method 
From synchrotrons for XFELs: the soft x-ray near-edge spectrum of the ESCA molecule8

[83] with a noniterative triples correction to EOM-CCSD, which has been shown to provide reasonable estimates for triples corrections to core excitation energies [52]. As shown in Table 1, the $\operatorname{EOM} \operatorname{CCSD}(\mathrm{T})(\mathrm{a})^{*}$ values for relative shifts between the $1 s \rightarrow \pi^{*}$ transition and the first Rydberg transition compare favorably with the EOMCCSDT values, although EOM-CCSD $(\mathrm{T})(\mathrm{a})^{*}$ underestimates the triples corrections to the absolute values of these core excitation energies by around $0.4 \mathrm{eV}$.

Table 1: Carbon and oxygen K-edge excitation energies $(\mathrm{eV})$ of formaldehyde. The relative shifts between the first Rydberg excitation $1 s \rightarrow 3 p\left(a_{1}\right)$ and the valence $1 s \rightarrow \pi^{*}$ transition $(\mathrm{eV})$ are enclosed in the parentheses. The cc-pVTZ $+5 \mathrm{spd}$ basis set was used for the targeted atom and cc-pVTZ basis sets for the other atoms.

Scalar-relativistic effects were taken into account using the spin-free exact two-component theory in its one-electron variant.

\begin{tabular}{ccccc}
\hline & \multicolumn{2}{c}{ carbon edge } & \multicolumn{2}{c}{ oxygen edge } \\
& $1 s \rightarrow \pi^{*}$ & $1 s \rightarrow 3 p\left(a_{1}\right)$ & $1 s \rightarrow \pi^{*}$ & $1 s \rightarrow 3 p\left(a_{1}\right)$ \\
\hline EOM-CCSD & 286.59 & $291.27(4.68)$ & 532.49 & $537.77(5.28)$ \\
EOM-CCSD(T)(a)* & 286.28 & $290.59(4.31)$ & 531.56 & $536.10(4.54)$ \\
EOM-CCSDT & 285.90 & $290.14(4.24)$ & 531.03 & $535.47(4.44)$ \\
Experiment [84] & 285.97 & $290.12(4.15)$ & 530.88 & $535.54(4.66)$ \\
\hline
\end{tabular}

Finally, computational NEXAFS spectra for the carbon edge of formaldehyde obtained using the EOM-CCSD(T)(a)* and CCSDT level positions and EOM-CCSD oscillator strength are presented in Figure 3. With basis-set effects on Rydberg coreexcited states taken into account using cc-pVTZ +5 spd basis and the inclusion of triples contributions, these computational NEXAFS spectra agree very well with corresponding experimental spectrum. Based on these benchmark calculations, the use of the $\operatorname{EOM-CCSD}(\mathrm{T})(\mathrm{a})^{*}$ method together with cc-pVTZ basis systematically extended with diffuse functions is expected to provide useful results for our present study of the ESCA molecule. 
From synchrotrons for XFELs: the soft x-ray near-edge spectrum of the ESCA molecule9

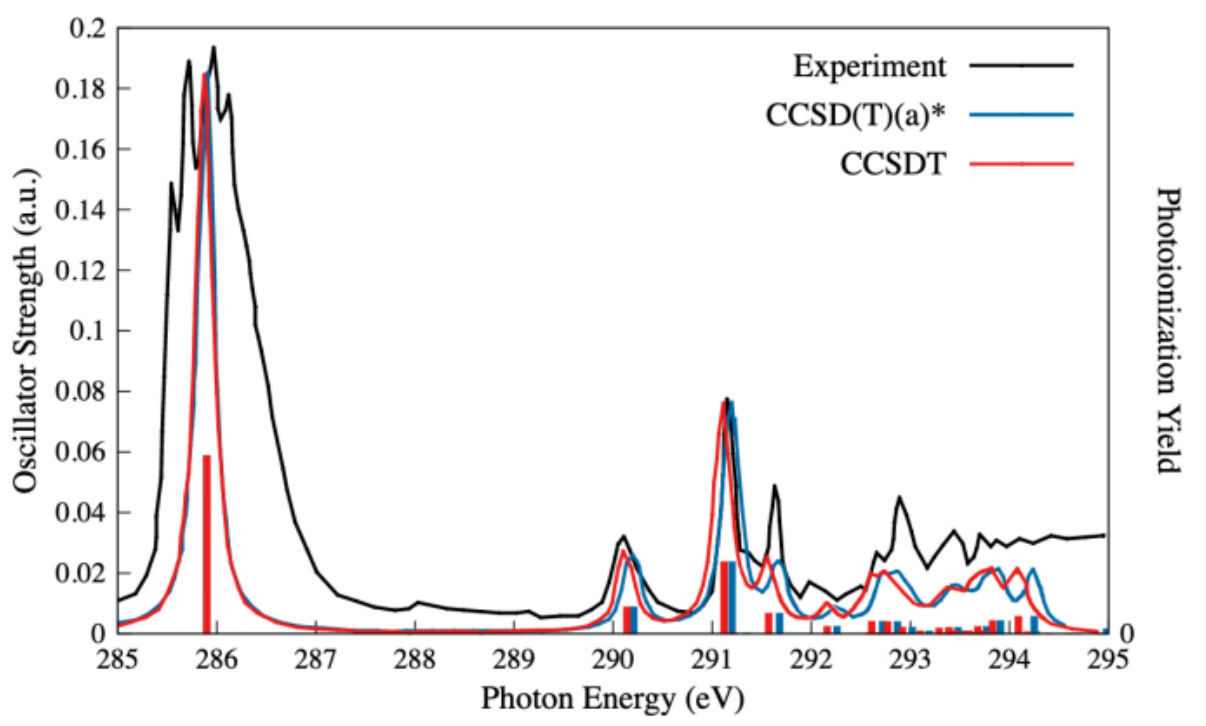

Figure 3: Experimental NEXAFS spectrum [84] for the carbon edge of formaldehyde together with computational spectra obtained using EOM-CCSDT and

EOM-CCSD $(\mathrm{T})(\mathrm{a})^{*}$ level positions and EOM-CCSD oscillator strength. Lorentzian functions with a FWHM value of $0.2 \mathrm{eV}$ have been used in the convolution of computational spectra. The $\operatorname{CCSD}(\mathrm{T})(\mathrm{a})^{*}$ level positions have been red-shifted by $0.38 \mathrm{eV}$. cc-pVTZ $+5 \mathrm{spd}$ basis sets have been used. Scalar-relativistic effects have

been taken into account using the spin-free exact two-component theory in its one-electron variant. Note that the finer structures of the experimental spectrum centered around $286 \mathrm{eV}$ originate from vibrational progression and hence are absent in the present simulation. [46]

\section{Results and discussion}

\subsection{Assignment of experimental NEXAFS spectrum using computational results}

Fig. 4 shows the computed NEXAFS spectrum for the four carbon edges of the ESCA molecule. The calculations have used the SFX2C-1e $\operatorname{EOM}-\operatorname{CCSD}(\mathrm{T})(\mathrm{a})^{*}$ method for transition energies, EOM-CCSD method for transition dipole moments, cc-pVTZ+4spd basis set for the targeted carbon, and cc-pVTZ basis sets for all other atoms. The density difference natural virtual orbital as plotted in Fig. 5-(A) shows that the first intense peak located at $288.80 \mathrm{eV}$ in the calculation corresponds to the carbonyl carbon $1 s \rightarrow \pi^{*}$ transition. Here this excitation into the valence $\pi^{*}$ orbital leads to a transition energy around $8 \mathrm{eV}$ lower than the 1s ionization energy of the carbonyl carbon, which lies in the middle of pre-edge transitions of the methyl carbon edge. Computed transition energies for $1 s \rightarrow \pi^{*}$ excitations in $\mathrm{CO}_{2}, \mathrm{CH}_{2} \mathrm{O}$, and the ESCA molecule are summarized in Table 2. The transition energies obtained from $\operatorname{EOM-CCSD}(\mathrm{T})(\mathrm{a})^{*} / \mathrm{cc}-\mathrm{pVTZ}+4 \mathrm{spd}$ calculations are consistently around $0.5 \mathrm{eV}$ higher than the corresponding experimental values, perhaps mainly due to the approximation in the treatment of triple excitations. The intense feature peaked at $288.31 \mathrm{eV}$ in the experimental spectrum can thus be confidently assigned to this carbonyl carbon 
From synchrotrons for XFELs: the soft x-ray near-edge spectrum of the ESCA molecule10

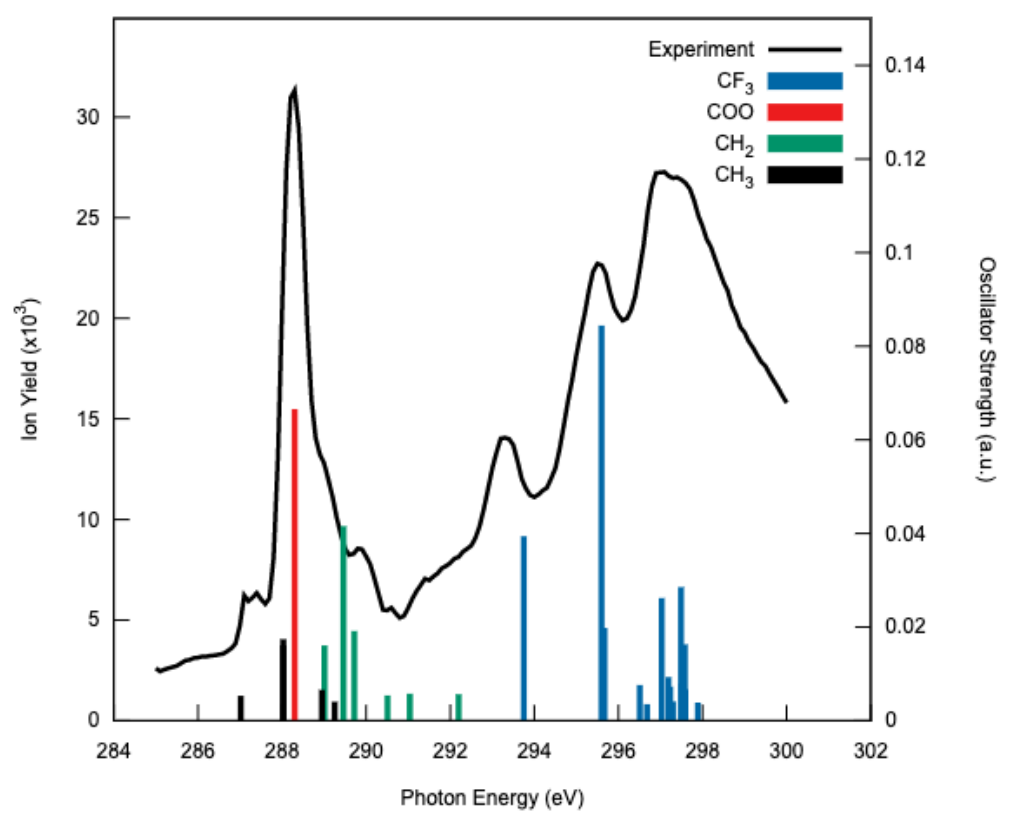

Figure 4: Experimental and computed NEXAFS spectra for the four carbon edges of the ethyl trifluoroacetate molecule. The black solid line shows the variation of measured ion yield with respect to photon energies. Blue, red, green, and black sticks represent computed absorption lines for the $\mathrm{CF}_{3}, \mathrm{COO}, \mathrm{CH}_{2}$, and $\mathrm{CH}_{3}$ carbon edges, respectively, with the height being the values of the computed oscillator strength. Energy level positions were computed using the $\operatorname{EOM}-\operatorname{CCSD}(\mathrm{T})(\mathrm{a})^{*}$ method, while transition dipole moments were obtained from EOM-CCSD calculations. The computed spectrum has been red-shifted by $0.50 \mathrm{eV}$ to align the computed $1 s \rightarrow \pi^{*}$ transition with the experiment. The corresponding unshifted spectrum is enclosed as

Fig. 1 of the supplementary material. Scalar-relativistic effects were taken into account using the spin-free exact two-component theory in its one-electron variant.

$1 s \rightarrow \pi^{*}$ transition. Further, in Fig. 4 we red-shifted all computed lines by $0.50 \mathrm{eV}$ to align the computed and experimental peaks for this transition. In the following discussions we will also use the red-shifted computed energies.

Table 2: Carbon $1 s \rightarrow \pi^{*}$ transition energies (eV). Scalar-relativistic effects were taken into account using the spin-free exact two-component theory in its one-electron variant.

\begin{tabular}{cccc}
\hline & $\mathrm{CO}_{2}$ & $\mathrm{CH}_{2} \mathrm{O}$ & ESCA \\
\hline EOM-CCSD/cc-pVTZ+4spd & 291.60 & 286.59 & 289.11 \\
EOM-CCSD(T) $(\mathrm{a})^{*} /$ cc-pVTZ+4spd & 291.33 & 286.28 & 288.80 \\
EOM-CCSDT/cc-pVTZ+4spd & 290.95 & 285.90 & $/$ \\
Experiment $[84,85]$ & 290.76 & 285.97 & 288.31 \\
\hline
\end{tabular}


From synchrotrons for XFELs: the soft x-ray near-edge spectrum of the ESCA molecule11

The first visible feature in the experimental spectrum, albeit a weak one, appears at round $287.02 \mathrm{eV}$ and is assigned to the methyl carbon $1 s \rightarrow \sigma^{*}$ transition [the corresponding DDNO is plotted in Fig. 5-(B)]. The computed transition energies for the $1 s \rightarrow 3 p$ transitions of the methyl carbon edge are very close to that of the carbonyl carbon $1 s \rightarrow \pi^{*}$ transition [Fig. 5 - $(\mathrm{C}, \mathrm{D})$ ]. The $1 s \rightarrow 4 p$ transitions are around $1 \mathrm{eV}$ higher and have much lower intensities. The transitions from 1s electron of the $\mathrm{CH}_{2}$ carbon to a C-H $\sigma^{*}$ orbital [Fig. 5-(E)], a C-O $\sigma^{*}$ orbital [Fig. 5-(F)], and the 3p orbitals [Fig. 5-(G)] are located at $289.0 \mathrm{eV}, 289.46 \mathrm{eV}$, and $289.73 \mathrm{eV}$, respectively. They also contribute to the intense feature centered around $288.3 \mathrm{eV}$. Interestingly, the most intense features from the $\mathrm{CH}_{3}, \mathrm{CH}_{2}$, and $\mathrm{COO}$ carbon edges all pile up in the pre-methyl carbon edge region ranging from $287 \mathrm{eV}$ to $291 \mathrm{eV}$. As mentioned in the introduction, this renders the NEXAFS spectrum of the ESCA molecule substantially more complicated than the corresponding photoelectron spectrum.

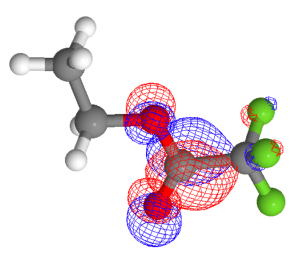

(A) COO $1 \mathrm{~s}->\pi^{*}$ (288.30 eV; 0.0664)

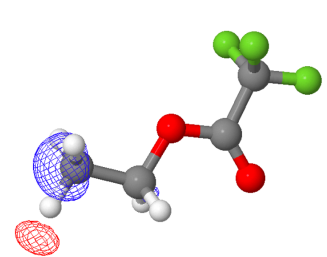

(B) $\mathrm{CH}_{3} 1 \mathrm{~s} \rightarrow \mathrm{C}-\mathrm{C} \boldsymbol{\sigma}^{*}$ (287.02 eV; 0.0051)

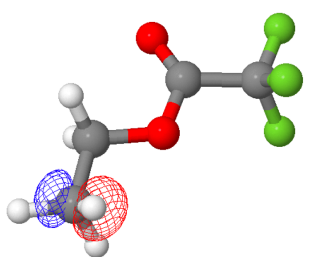

(C) $\mathrm{CH}_{3} 1 \mathrm{~s}->3 \mathrm{p}$ (288.03 eV; 0.0172)

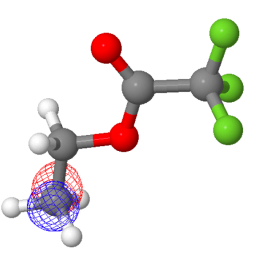

(D) $\mathrm{CH}_{3} 1 \mathrm{~s}->3 \mathrm{p}$ (288.03 eV; 0.0158)

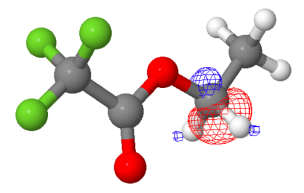

(E) $\mathrm{CH}_{2}$ 1s $\rightarrow \mathrm{C}-\mathrm{H} \sigma^{*}$ (289.01 eV; 0.0159)

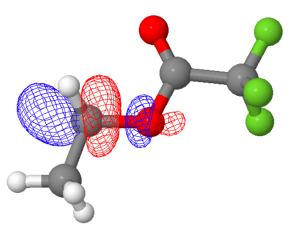

(F) $\mathrm{CH}_{2} 1 \mathrm{~s}->\mathrm{C}-\mathrm{O} \sigma^{*}$ (289.46 eV; 0.0414)

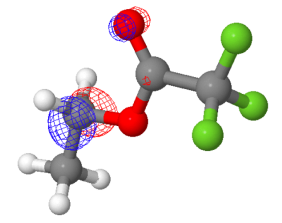

(G) $\mathrm{CH}_{2} 1 \mathrm{~s}->3 \mathrm{p}$ (289.72 eV; 0.0190)

Figure 5: Density-difference natural virtual orbitals for core-excited states of COO, $\mathrm{CH}_{3}$, and $\mathrm{CH}_{2} \mathrm{C}$ edges of the ESCA molecule that are the most intense ranging from $287 \mathrm{eV}$ to $292 \mathrm{eV}$ in the NEXAFS spectrum. Computed transition energies (with a $0.5 \mathrm{eV}$ red shift) and oscillator strengths as used in Fig. 4 are enclosed in the parentheses.

The features in the experimental spectrum with transition energies higher than $293 \mathrm{eV}$ are assigned to transitions of the $\mathrm{CF}_{3}$ carbon edge. The transition around 293.5 $\mathrm{eV}$ is a valence excitation into a $\mathrm{C}-\mathrm{C} \pi$ orbital, as shown in Fig. 6-(H). Interestingly, since the electron density between the two carbon atoms is heavily depleted due to the presence of three fluorine atoms with high electronegativity, this excitation that increases the electron density in an orbital of bonding character in this region produces an excited state with substantially lower energy than excitations to anti-bonding or Rydberg orbitals. The strong transition around $295.8 \mathrm{eV}$ in the experimental spectrum is assigned to a valence excitation into the $\mathrm{C}-\mathrm{F} \sigma^{*}$ orbital, which is calculated to be 
located at $296.14 \mathrm{eV}$ [Fig. 6-(I)]. The broad feature centered around $297 \mathrm{eV}$ consists of Rydberg transitions to 3p [Fig. 6-(J, K, L)] and 4p orbitals.

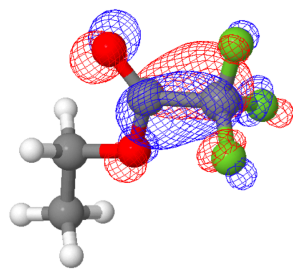

(H) $\mathrm{CF}_{3} 1 \mathrm{~s}->\mathrm{C}-\mathrm{C} \pi$ (293.75 eV; 0.0392)

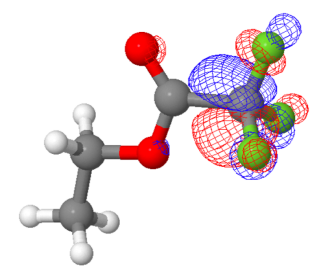

(I) $\mathrm{CF}_{3}$ 1s $\rightarrow \mathrm{C}-\mathrm{F} \boldsymbol{\sigma}^{*}$ (295.60 eV; 0.0842)

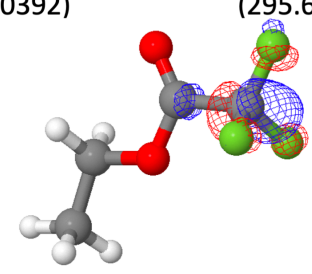

(K) $\mathrm{CF}_{3} 1 \mathrm{~s}->3 \mathrm{p}$

(297.03 eV; 0.0260)

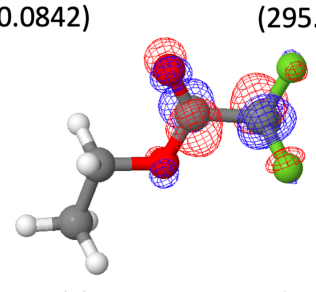

(L) $\mathrm{CF}_{3} 1 \mathrm{~s}->3 \mathrm{p}+\pi^{*}$

(297.49 eV; 0.0283)

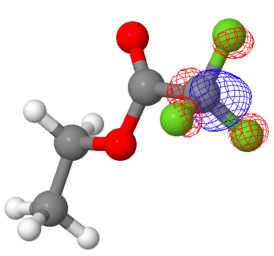

(J) $\mathrm{CF}_{3} 1 \mathrm{~s}->3 \mathrm{p}$

(295.67 eV; 0.0196)

Figure 6: Density-difference natural virtual orbitals for intense core excited states of the $\mathrm{CF}_{3}$ carbon edge of the ESCA molecule that are the most intense in the range of 292-299 eV in the NEXAFS spectrum. Computed transition energies (with a $0.5 \mathrm{eV}$ red shift) and oscillator strengths as used in Fig. 4 are enclosed in the parentheses.

\subsection{Remarks on the accuracy of the computed spectrum}

Since computations play an important role in the assignment of the experimental spectrum, it is of interest to analyze the accuracy of the present computational results in more detail. First we focus on the description of core-excited Rydberg states. The use of the cc-pVTZ+4spd basis set for the target carbon can describe three sets of Rydberg states accurately, which is expected to cover important features in the experimental NEXAFS spectrum. As shown in Fig. 2-4 in the supplementary material, the cc-pVTZ +2 spd basis provides an essentially converged description for valence excitations and the first sets of Rydberg excitations. However, inclusion of additional diffuse functions in the cc-pVTZ+3spd and cc-pVTZ+4spd sets provides richer structures and smaller intensities for higher Rydberg states, which clearly improves the quality of the simulated spectrum. Inspection of the convergence of computational results shows that further addition of a fifth set of diffuse functions is unlikely to make a significant difference. It can thus be safely concluded that the treatment of the diffuse character of Rydberg states has been converged in the calculations using the cc-pVTZ+4spd basis set. Note that the augmentation of standard basis sets with sufficient diffuse functions is required to capture the diffuse nature of core-excited Rydberg states in general. In recent time-dependent density-functional theory (TDDFT) and restricted active space second order perturbation theory (RASPT2) calculations of the NEXAFS spectrum for the ESCA molecule [20], the use of standard basis sets also seem to lead 
to overestimation of intensities for Rydberg states. The present edge-specific approach is expected to be applicable for quantum-chemical methods other than coupled-cluster methods in a straightforward manner.

Accurate calculations of transition energies in the NEXAFS spectra has emerged as a major challenge. The experimental NEXAFS spectrum for the ESCA molecule prensented here serves as a useful guide for benchmarking computational methods. Comparing the experimental and computational spectra, it appears that an accurate calculation for the separation between the $1 s \rightarrow \pi^{*}$ transition of the carbonyl carbon and other transitions is a formidable task. Triples corrections clearly make important contributions here. Comparing the CCSD/cc-pVTZ+4spd spectrum (Fig. 4 of the supplementary material) and the $\operatorname{CCSD}(\mathrm{T})(\mathrm{a})^{*} /$ cc-pVTZ+4spd spectrum (Fig. 4), it can be seen that CCSD clearly overestimates the separation between the carbonyl carbon $1 s \rightarrow \pi^{*}$ transition and the other transitions with higher energies. For example, the triples corrections red-shift the level positions of the transitions around $298 \mathrm{eV}$ in the spectrum by around $1 \mathrm{eV}$. A particularly difficult case is the separation between the carbonyl carbon $1 s \rightarrow \pi^{*}$ transition and the $\mathrm{CH}_{2}$ carbon $1 s$ to C-O $\sigma^{*}$ transition, which amounts to $1.41 \mathrm{eV}$ at the CCSD level. The inclusion of triples corrections using $\operatorname{CCSD}(\mathrm{T})(\mathrm{a})^{*}$ reduces this value to $1.16 \mathrm{eV}$. However, even $\operatorname{CCSD}(\mathrm{T})(\mathrm{a})^{*}$ seems to overestimate this separation. In the experimental spectrum, only a small shoulder appears around $0.7 \mathrm{eV}$ higher than the peak at $288.30 \mathrm{eV}$, which may indicate that the strong transition from the $\mathrm{CH}_{2}$ carbon $1 s$ orbital to the $\mathrm{C}-\mathrm{O} \sigma^{*}$ orbital is located even closer to the carbonyl carbon $1 s \rightarrow \pi^{*}$ transition, so that both of them contribute to the intense peak at $288.3 \mathrm{eV}$. This difficulty also persists for other computational methods. The TDDFT and RASPT2 calculations reported in Ref. [20] also successfully identified the carbonyl carbon $1 s \rightarrow \pi^{*}$ transition as the first intense transition. However, the separation between the carbonyl carbon $1 s \rightarrow \pi^{*}$ transition and the $\mathrm{CH}_{2}$ carbon $1 s$ to $\mathrm{C}-\mathrm{O} \sigma^{*}$ transition has also been overestimated, with the TDDFT value being $1.5 \mathrm{eV}$ and the RASSCF/RASPT2 value being as large as $c a .3 \mathrm{eV}$. It would be of interest to further investigate the calculations of these transition energies. In particular, delta-coupled-cluster methods, which directly calculate coupled-cluster wavefunctions for core excited states [77, 86, 52], may be able to provide more accurate energies for these states than the CVS-EOM-CC methods with approximate treatment of triple excitations. Further, vibronic coupling has been reported to make significant contributions to NEXAFS of $\mathrm{CO}_{2}[85]$ and might be relevant to the ESCA molecule. This, however, is only speculation at the moment.

The sum of oscillator strengths for transitions from $\mathrm{CF}_{3}, \mathrm{COO}, \mathrm{CH}_{2}$, and $\mathrm{CH}_{3}$ edges are $0.277,0.075,0.108$, and 0.066 . The $\mathrm{CF}_{3}$ edge has substantially higher intensity for core excitations than other edges, perhaps because the depletion of the electron density around this carbon and the $\mathrm{sp}^{3}$ hybridization of this carbon atom offers good accommodation of various valence and Rydberg excitations. The present simulation has not considered contributions from core ionizations to the intensities of the absorption spectrum. Although the main peaks in the absorption spectrum seem to originate from core excitations and core ionizations likely contribute as smooth background beyond each edge, it would be of interest to investigate the contributions from core ionization using the Dyson orbital technique $[87,88,89,49]$ to get a complete description for the absorption spectrum. We should also mention that CVS-EOM$\mathrm{CC}$ methods used here work with wavefunctions of bound states and are suitable 
From synchrotrons for XFELs: the soft x-ray near-edge spectrum of the ESCA molecule14

for obtaining valence excitations and Rydberg excitations series that systematically approach the ionization threshold. They may not be suitable for describing states beyond the ionization threshold, which in general requires the use of the so-called "non-Hermitian quantum mechanics" [90, 91]. Therefore, we only show results for core excited states with excitation energies below the ionization energy for every carbon edge in present discussions.

\section{Summary and Outlook}

We present the experimental photoabsorption spectrum of the ESCA molecule, ethyl trifluoroacetate, near the carbon K-edge and provide a theoretical interpretation of its spectral features. The theoretical methods advance the theoretical state-of-the-art by saturating basis-set effects to describe properly the diffuse Rydberg orbitals using a simple edge-specific approach for systematically expanding standard basis sets, and, by including triple excitations to capture the valence (in particular $1 s \rightarrow \pi^{*}$ )-to-Rydberg energy intervals. These methods were benchmarked on other simple molecules $\left(\mathrm{NH}_{3}\right.$, $\mathrm{H}_{2} \mathrm{CO}, \mathrm{CO}_{2}$ ) and are thought to be generalizable. While most time-resolved NEXAFS spectra have been used to interpret dynamics due to optical/UV excitation of valence states [26, 16], NEXAFS can also provide fingerprints to follow inner-shell-initiated molecular dynamics - a discipline which is now feasible and a natural extension to x-ray pump/probe methods at XFELs [17, 92, 93, 94, 95]. Ethyl trifluoroacetate has already proven itself a model for ESCA (electron spectroscopy for chemical analysis), and, now extends its reach to advance theoretical methods to describe complex innershell photoabsorption spectra.

\section{Acknowledgements}

This work was primarily supported by the U.S. Department of Energy, Office of Basic Energy Sciences, Division of Chemical Sciences, Geosciences, and Biosciences through Argonne National Laboratory. Argonne is a U.S. Department of Energy laboratory managed by UChicago Argonne, LLC, under contract DE-AC02-06CH11357. SLS acknowledges support from MAX4ESSFUN within the EU inter-reg project ÖKS (ESS MAX IV: Cross Border Science and Society). M.P. and E.K. acknowledge support from Academy of Finland. Experiments were performed on the PLEIADES beamline at SOLEIL Synchrotron, France (Proposal No. 20160380). We are grateful to the SOLEIL staff for smoothly running the facility.

\section{References}

[1] Emma P, Akre R, Arthur J, Bionta R, Bostedt C, Bozek J, Brachmann A, Bucksbaum P, Coffee R, Decker F J et al. 2010 Nature Photonics 4641

[2] Ishikawa T, Aoyagi H, Asaka T, Asano Y, Azumi N, Bizen T, Ego H, Fukami K, Fukui T, Furukawa Y, Goto S, Hanaki H, Hara T, Hasegawa T, Hatsui T, Higashiya A, Hirono T, Hosoda N, Ishii M, Inagaki T, Inubushi Y, Itoga T, Joti Y, Kago M, Kameshima T, Kimura H, Kirihara Y, Kiyomichi A, Kobayashi T, Kondo C, Kudo T, Maesaka H, Maréchal X M, Masuda T, Matsubara S, Matsumoto T, Matsushita T, Matsui S, Nagasono M, Nariyama N, Ohashi H, Ohata T, Ohshima T, Ono S, Otake Y, Saji C, Sakurai T, Sato T, Sawada K, Seike T, Shirasawa K, Sugimoto T, Suzuki S, Takahashi S, Takebe H, Takeshita K, Tamasaku K, Tanaka H, Tanaka R, Tanaka T, Togashi T, Togawa K, Tokuhisa A, Tomizawa H, Tono K, Wu S, Yabashi M, Yamaga M, Yamashita A, Yanagida K, Zhang C, Shintake T, Kitamura H and Kumagai N 2012 Nature Photonics 6 540-544 
From synchrotrons for XFELs: the soft x-ray near-edge spectrum of the ESCA molecule15

[3] Allaria E, Badano L, Bassanese S, Capotondi F, Castronovo D, Cinquegrana P, Danailov M B, D'Auria G, Demidovich A, De Monte R, De Ninno G, Di Mitri S, Diviacco B, Fawley W M, Ferianis M, Ferrari E, Gaio G, Gauthier D, Giannessi L, Iazzourene F, Kurdi G, Mahne N, Nikolov I, Parmigiani F, Penco G, Raimondi L, Rebernik P, Rossi F, Roussel E, Scafuri C, Serpico C, Sigalotti P, Spezzani C, Svandrlik M, Svetina C, Trovó M, Veronese M, Zangrando D and Zangrando M 2015 Journal of Synchrotron Radiation 22 485-491

[4] Ko I, Kang H S, Heo H, Kim C, Kim G, Min C K, Yang H, Baek S, Choi H J, Mun G and et al 2017 Applied Sciences $\mathbf{7} 479$

[5] Milne C, Schietinger T, Aiba M, Alarcon A, Alex J, Anghel A, Arsov V, Beard C, Beaud P, Bettoni S and et al 2017 Applied Sciences 7720

[6] Decking W, Abeghyan S, Abramian P, Abramsky A, Aguirre A, Albrecht C, Alou P, Altarelli M, Altmann P, Amyan K et al. 2020 Nature Photonics 1-7

[7] Schoenlein R, Elsaesser T, Holldack K, Huang Z, Kapteyn H, Murnane M and Woerner M 2019 Philosophical Transactions of the Royal Society A: Mathematical, Physical and Engineering Sciences 37720180384

[8] Popmintchev T, Chen M C, Popmintchev D, Arpin P, Brown S, Ališauskas S, Andriukaitis G, Balčiunas T, Mücke O D, Pugzlys A, Baltuška A, Shim B, Schrauth S E, Gaeta A, HernándezGarcía C, Plaja L, Becker A, Jaron-Becker A, Murnane M M and Kapteyn H C 2012 Science 336 1287-1291

[9] Young L, Ueda K, Gühr M, Bucksbaum P H, Simon M, Mukamel S, Rohringer N, Prince K C, Masciovecchio C, Meyer M, Rudenko A, Rolles D, Bostedt C, Fuchs M, Reis D A, Santra R, Kapteyn H, Murnane M, Ibrahim H, Légaré F, Vrakking M, Isinger M, Kroon D, Gisselbrecht M, L'Huillier A, Wörner H J and Leone S R 2018 Journal of Physics B: Atomic, Molecular and Optical Physics $\mathbf{5 1} 032003$

[10] Marinelli A, MacArthur J, Emma P, Guetg M, Field C, Kharakh D, Lutman A A, Ding Y and Huang Z 2017 Applied Physics Letters 111151101

[11] Huang S, Ding Y, Feng Y, Hemsing E, Huang Z, Krzywinski J, Lutman A A, Marinelli A, Maxwell T J and Zhu D 2017 Phys. Rev. Lett. 119(15) 154801

[12] Duris J, Li S, Driver T, Champenois E G, MacArthur J P, Lutman A A, Zhang Z, Rosenberger P, Aldrich J W, Coffee R et al. 2020 Nature Photonics 14 30-36

[13] Lutman A A, Coffee R, Ding Y, Huang Z, Krzywinski J, Maxwell T, Messerschmidt M and Nuhn H D 2013 Phys. Rev. Lett. 110(13) 134801

[14] Marinelli A, Ratner D, Lutman A A, Turner J, Welch J, Decker F J, Loos H, Behrens C, Gilevich S, Miahnahri A A, Vetter S, Maxwell T J, Ding Y, Coffee R, Wakatsuki S and Huang Z 2015 Nature Communications 66369

[15] Lutman A A, Maxwell T J, MacArthur J P, Guetg M W, Berrah N, Coffee R N, Ding Y, Huang Z, Marinelli A, Moeller S et al. 2016 Nature Photonics 10 745-750

[16] Wolf T J A, Myhre R H, Cryan J P, Coriani S, Squibb R J, Battistoni A, Berrah N, Bostedt C, Bucksbaum P, Coslovich G, Feifel R, Gaffney K J, Grilj J, Martinez T J, Miyabe S, Moeller S P, Mucke M, Natan A, Obaid R, Osipov T, Plekan O, Wang S, Koch H and Gühr M 2017 Nature Communications $\mathbf{8} 29$

[17] Picón A, Lehmann C S, Bostedt C, Rudenko A, Marinelli A, Osipov T, Rolles D, Berrah N, Bomme C, Bucher M, Doumy G, Erk B, Ferguson K R, Gorkhover T, Ho P J, Kanter E P, Krässig B, Krzywinski J, Lutman A A, March A M, Moonshiram D, Ray D, Young L, Pratt S T and Southworth S H 2016 Nature Communications 711652

[18] Neville S P, Averbukh V, Patchkovskii S, Ruberti M, Yun R, Chergui M, Stolow A and Schuurman M S 2016 Faraday Discuss. 194(0) 117-145

[19] Mukamel S, Healion D, Zhang Y and Biggs J D 2013 Annu. Rev. Phys. Chem. 64 101-127

[20] Nenov A, Segatta F, Bruner A, Mukamel S and Garavelli M 2019 J. Chem. Phys. 151114110

[21] Siegbahn K 1967 Nova Acta Regiae Societatis Scientiarum Upsaliensis

[22] Siegbahn K 1970 ESCA applied to free molecules (North-Holland Pub. Co.)

[23] Travnikova O, Børve K J, Patanen M, Söderström J, Miron C, Sæthre L J, Mårtensson N and Svensson S 2012 Journal of Electron Spectroscopy and Related Phenomena 185191 - 197 special Issue in honor of Prof. T. Darrah Thomas: High-Resolution Spectroscopy of Isolated Species

[24] Inhester L, Oostenrijk B, Patanen M, Kokkonen E, Southworth S H, Bostedt C, Travnikova O, Marchenko T, Son S K, Santra R, Simon M, Young L and Sorensen S L 2018 J. Chem. Phys. Lett. 9 1156-1163

[25] Stöhr J 2003 NEXAFS Spectroscopy (Springer-Verlag Berlin Heidelberg New York)

[26] Bhattacherjee A and Leone S R 2018 Accounts of Chemical Research 51 3203-3211

[27] Pertot Y, Schmidt C, Matthews M, Chauvet A, Huppert M, Svoboda V, von Conta A, Tehlar 
From synchrotrons for XFELs: the soft x-ray near-edge spectrum of the ESCA molecule16

A, Baykusheva D, Wolf J P and Wörner H J 2017 Science 355 264-267

[28] Wang H Y, Schreck S, Weston M, Liu C, Ogasawara H, LaRue J, Perakis F, Dell'Angela M, Capotondi F, Giannessi L, Pedersoli E, Naumenko D, Nikolov I, Raimondi L, Spezzani C, Beye M, Cavalca F, Liu B, Gladh J, Koroidov S, Miedema P S, Costantini R, Pettersson L G M and Nilsson A 2020 Phys. Chem. Chem. Phys. 22 2677-2684

[29] Northey T, Norell J, Fouda A E A, Besley N A, Odelius M and Penfold T J 2020 Phys. Chem. Chem. Phys. 22 2667-2676

[30] Norell J, Eckert S, Van Kuiken B E, Föhlisch A and Odelius M 2019 J. Chem. Phys. 151114117

[31] Norman P and Dreuw A 2018 Chem. Rev. 118 7208-7248

[32] Besley N A 2020 Acc. Chem. Res. 53 1306-1315

[33] Stanton J F and Bartlett R J 1993 J. Chem. Phys. 98 7029-7039

[34] Krylov A I 2008 Ann. Rev. Phys. Chem. 59 433-462

[35] Besley N A 2012 Chem. Phys. Lett. 542 42-46

[36] Coriani S, Christiansen O, Fransson T and Norman P 2012 Phys. Rev. A 85022507

[37] Southworth S H, Wehlitz R, Picón A, Lehmann C S, Cheng L and Stanton J F 2015 J. Chem. Phys. 142224302

[38] Peng B, Lestrange P J, Goings J J, Caricato M and Li X 2015 J. Chem. Theory Comput. 11 $4146-4153$

[39] Nascimento D R and Deprince A E 2017 J. Phys. Chem. Lett. 8 2951-2957

[40] Cederbaum L S, Domcke W and Schirmer J 1980 Phys. Rev. A At., Mol., Opt. Phys. 22 206-222

[41] Coriani S and Koch H 2015 J. Chem. Phys. 143181103

[42] Myhre R H, Coriani S and Koch H 2016 J. Chem. Theory Comput. 12 2633-2643

[43] Yang Z, Schnorr K, Bhattacherjee A, Lefebvre P L, Epshtein M, Xue T, Stanton J F and Leone S R 2018 J. Am. Chem. Soc. 140 13360-13366

[44] Vidal M L, Feng X, Epifanovsky E, Krylov A I and Coriani S 2019 J. Chem. Theory Comput. $153117-3133$

[45] Liu J, Matthews D, Coriani S and Cheng L 2019 J. Chem. Theory Comput. 15 1642-1651

[46] Frati F, De Groot F, Cerezo J, Santoro F, Cheng L, Faber R and Coriani S 2019 J. Chem. Phys. 151064107

[47] Tenorio B N C, Moitra T, Nascimento M A C, Rocha A B and Coriani S 2019 J. Chem. Phys. 150224104

[48] Park Y C, Perera A and Bartlett R J 2019 J. Chem. Phys. 151164117

[49] Vidal M L, Krylov A I and Coriani S 2020 Phys. Chem. Chem. Phys. 22 2693-2703

[50] Faber R and Coriani S 2020 Phys. Chem. Chem. Phys. 22 2642-2647

[51] Nanda K D, Vidal M L, Faber R, Coriani S and Krylov A I 2020 Phys. Chem. Chem. Phys. 22 $2629-2641$

[52] Matthews D A 2020 Mol. Phys. 0 1-8

[53] Bazante A P, Perera A and Bartlett R J 2017 Chem. Phys. Lett. 683 68-75

[54] Myhre R H, Coriani S and Koch H 2019 J. Phys. Chem. A 123 9701-9711

[55] Tsuru S, Vidal M L, Pápai M, Krylov A I, Møller K B and Coriani S 2019 J. Chem. Phys. 151 124114

[56] Kjellsson L, Nanda K D, Rubensson J E, Doumy G, Southworth S H, Ho P J, March A M, Al Haddad A, Kumagai Y, Tu M F, Schaller R D, Debnath T, Bin Mohd Yusof M S, Arnold C, Schlotter W F, Moeller S, Coslovich G, Koralek J D, Minitti M P, Vidal M L, Simon M, Santra R, Loh Z H, Coriani S, Krylov A I and Young L 2020 Phys. Rev. Lett. 124236001

[57] Myhre R H, Wolf T J, Cheng L, Nandi S, Coriani S, Gühr M and Koch H 2018 J. Chem. Phys. 148064106

[58] Southworth S H, Dunford R W, Ray D, Kanter E P, Doumy G, March A M, Ho P J, Krässig B, Gao Y, Lehmann C S, Picón A, Young L, Walko D A and Cheng L 2019 Phys. Rev. A 100 22507

[59] Fano U and Cooper J W 1968 Rev. Mod. Phys. 40(3) 441-507

[60] Dehmer J L and Dill D 1975 Phys. Rev. Lett. 35(4) 213-215

[61] Becker U and Shirley D A E 1996 VUV and Soft X-ray Photoionization (Plenum Press, New York and London)

[62] Young L, Kanter E P, Krässig B, Li Y, March A M, Pratt S T, Santra R, Southworth S H, Rohringer N, DiMauro L F, Doumy G, Roedig C A, Berrah N, Fang L, Hoener M, Bucksbaum P H, Cryan J P, Ghimire S, Glownia J M, Reis D A, Bozek J D, Bostedt C and Messerschmidt M 2010 Nature 466 56-61

[63] Santra R and Young L 2015 Interaction of intense x-ray beams with atoms Synchrotron Light Sources and Free-Electron Lasers ed Jaeschke E, Khan S, Schneider J and Hastings J (Springer International Publishing) pp 1-24 
From synchrotrons for XFELs: the soft x-ray near-edge spectrum of the ESCA molecule17

[64] Ueda K 2003 Journal of Physics B: Atomic, Molecular and Optical Physics 36 R1-R47

[65] Miron C, Simon M, Leclercq N and Morin P 1997 Review of Scientific Instruments 68 3728-3737

[66] Liu X J, Nicolas C, Robert E and Miron C 2014 Journal of Physics: Conference Series 488 142005

[67] Nicolas C and Miron C 2012 Journal of Electron Spectroscopy and Related Phenomena 185 $267-272$

[68] Adachi J i, Kosugi N, Shigemasa E and Yagishita A 1996 J. Phys. Chem. 100 19783-19788

[69] Dyall K G 2001 J. Chem. Phys. 115 9136-9143

[70] Liu W and Peng D 2009 J. Chem. Phys. 131 1-5

[71] Woon D E and Dunning, Jr T H 1995 J. Chem. Phys. 103 4572-4585

[72] Matthews D A, Cheng L, Harding M E, Lipparini F, Stopkowicz S, Jagau T C, Szalay P G, Gauss J and Stanton J F 2020 J. Chem. Phys. 152214108

[73] Stanton J F, Gauss J, Cheng L, Harding M E, Matthews D A and Szalay P G CFOUR, CoupledCluster techniques for Computational Chemistry, a quantum-chemical program package with contributions from A.A. Auer, R.J. Bartlett, U. Benedikt, C. Berger, D.E. Bernholdt, Y.J. Bomble, O. Christiansen, F. Engel, R. Faber, M. Heckert, O. Heun, M. Hilgenberg, C. Huber, T.-C. Jagau, D. Jonsson, J. Jusélius, T. Kirsch, K. Klein, W.J. Lauderdale, F. Lipparini, T. Metzroth, L.A. Mück, D.P. O'Neill, D.R. Price, E. Prochnow, C. Puzzarini, K. Ruud, F. Schiffmann, W. Schwalbach, C. Simmons, S. Stopkowicz, A. Tajti, J. Vázquez, F. Wang, J.D. Watts and the integral packages MOLECULE (J. Almlöf and P.R. Taylor), PROPS (P.R. Taylor), ABACUS (T. Helgaker, H.J. Aa. Jensen, P. Jørgensen, and J. Olsen), and ECP routines by A. V. Mitin and C. van Wüllen. For the current version, see http://www.cfour.de.

[74] Cheng L and Gauss J 2011 J. Chem. Phys. 135084114

[75] Matthews D A, Gauss J and Stanton J F 2013 J. Chem. Theory Comput. 9 2567-2572

[76] Matthews D A and Stanton J F 2015 J. Chem. Phys. 142064108

[77] Zheng X and Cheng L 2019 J. Chem. Theory Comput. 15 4945-4955

[78] Krylov A I 2020 J. Chem. Phys. 15380901

[79] Head-Gordon M, Grana A M, Maurice D and White C A 1995 J. Phys. Chem. 99 14261-14270

[80] Schirmer J, Trofimov A B, Randall K J, Feldhaus J, Bradshaw A M, Ma Y, Chen C T and Sette F 1993 Phys. Rev. A 47(2) 1136-1147

[81] Kowalski K and Piecuch P 2001 J. Chem. Phys. 115 643-651

[82] Kucharski S A, Włoch M, Musiał M and Rodney J Bartlett R 2001 J. Chem. Phys. 115 82638266

[83] Matthews D A and Stanton J F 2016 J. Chem. Phys. 145124102

[84] Remmers G, Domke M, Puschmann A, Mandel T, Xue C, Kaindl G, Hudson E and Shirley D A 1992 Phys. Rev. A 46(7) 3935-3944

[85] Prince K C, Avaldi L, Coreno M, Camilloni R and de Simone M 1999 Journal of Physics B: Atomic, Molecular and Optical Physics 32 2551-2567

[86] Lee J, Small D W and Head-Gordon M 2019 J. Chem. Phys. 151214103

[87] Fujikawa T 1982 J. Phys. Soc. Japan 51 2619-2627

[88] Lindgren I 2004 J. Electron Spectros. Relat. Phenomena 137-140 59-71

[89] Neville S P, Averbukh V, Ruberti M, Yun R, Patchkovskii S, Chergui M, Stolow A and Schuurman M S 2016 J. Chem. Phys. 145144307

[90] Moiseyev N 2011 Non-Hermitian Quantum Mechanics (Cambridge: Cambridge Univ. Press)

[91] Jagau T C, Bravaya K B and Krylov A I 2017 Annu. Rev. Phys. Chem. 68 525-553

[92] Zheng X, Liu J, Doumy G, Young L and Cheng L 2020124 4413-4426

[93] Liekhus-Schmaltz C E, Tenney I, Osipov T, Sanchez-Gonzalez A, Berrah N, Boll R, Bomme C, Bostedt C, Bozek J D, Carron S et al. 2015 Nature Communications 6 1-7

[94] Oberli S, González-Vázquez J, Rodríguez-Perelló E, Sodupe M, Martín F and Picón A 2019 Phys. Chem. Chem. Phys. 21 25626-25634

[95] Cooper B, Kolorenč P, Frasinski L J, Averbukh V and Marangos J P 2014 Faraday discussions $17193-111$ 


\title{
From synchrotrons for XFELs: the soft x-ray near-edge spectrum of the ESCA molecule - Supplementary Material
}

\author{
S.L. Sorensen ${ }^{1}$, X. Zheng ${ }^{2}$, S.H. Southworth ${ }^{3}$, M. Patanen ${ }^{4}$, \\ E. Kokkonen ${ }^{4,5}$, B. Oostenrijk ${ }^{1}$, O. Travnikova ${ }^{6}$, T. \\ Marchenko $^{6}$, M. Simon ${ }^{6}$, C. Bostedt ${ }^{3,7,8}$, G. Doumy ${ }^{3}$, L. \\ Cheng $^{2}$, L. Young ${ }^{3,9}$ \\ ${ }^{1}$ Department of Physics, Lund University, Box 118, 22100 Lund, Sweden \\ ${ }^{2}$ Department of Chemistry, Johns Hopkins University, Baltimore USA \\ ${ }^{3}$ Chemical Sciences and Engineering Division, Argonne National Laboratory, \\ 9700 S Cass Avenue, Lemont, IL 60439, United States of America \\ ${ }^{4}$ Faculty of Science, Nano and Molecular Systems Research Unit, University of \\ Oulu, Box 3000, FIN-90014 Oulu, Finland \\ ${ }^{5}$ MAX IV Laboratory, Lund University, Box 118, SE-221 00 Lund, Sweden \\ ${ }^{6}$ Sorbonne Université, CNRS, Laboratoire de Chimie Physique - Matière et \\ Rayonnement, LCPMR, F-75005 Paris, France \\ ${ }^{7}$ Paul Scherrer Institut, Switzerland \\ ${ }^{8}$ LUXS Laboratory for Ultrafast X-ray Sciences, École Polytechnique Fédérale \\ de Lausanne, Switzerland \\ ${ }^{9}$ Department of Physics and James Franck Institute, The University of Chicago, \\ Chicago, 60637, USA \\ E-mail: young@anl.gov
}

Figure 1 in the supplementary material is the unshifted version of Figure 3 in the main text. The original $\operatorname{EOM}-\operatorname{CCSD}(\mathrm{T})(\mathrm{a})^{*}$ energies were used here. In figure 3 of the main text, the lines are red-shifted by $0.5 \mathrm{eV}$ to align the computed COO $1 s \rightarrow \pi^{*}$ transition with the peak at $288.3 \mathrm{eV}$.

Figure 2, 3, and 4 in the supplementary material are EOM-CCSD spectrum obtained using cc-pVTZ +2 spd, cc-pVTZ +3 spd, and cc-pVTZ +4 spd basis for targeted atoms. The purpose is to show the convergence of the description for Rydberg states to the effects due to diffuse functions. The cc-pVTZ +2 spd has correct description for local excitations and the first set of Rydberg states, but overestimate energies and intensities for higher Rydberg transitions, with transitions around $298 \mathrm{eV}$ as prominent examples.

Table 1 in the supplementary material summarizes the raw data for figure 3 in the main text.

Table 2 in the supplementary material presents the raw data for figure 2 in the main text. 
From synchrotrons for XFELs: the soft x-ray near-edge spectrum of the ESCA molecule — Supplementary Material2

Table 3 in the supplementary material presents a comparison of computational results for the ESCA molecule in the $\mathrm{C}_{s}$ geometry with those in $\mathrm{C}_{1}$ geometry. 
From synchrotrons for XFELs: the soft x-ray near-edge spectrum of the ESCA molecule — Supplementary Material3

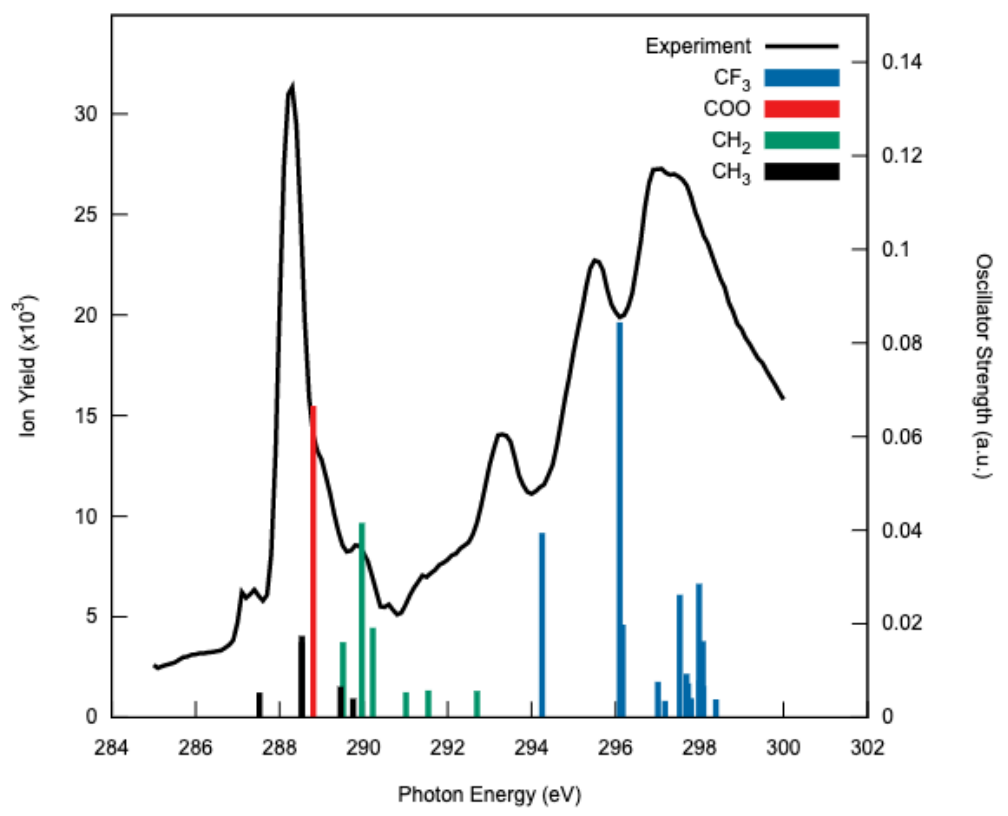

Figure 1: Experimental and computed NEXAFS spectra for the carbon edges of the ethyl trifluoroacetate molecule. The black solid line shows the variation of measured ion yield with respect to photon energies. Blue, red, green, and black sticks represent computed absorption lines for the $\mathrm{CF}_{3}, \mathrm{COO}, \mathrm{CH}_{2}$, and $\mathrm{CH}_{3}$ carbon edges, respectively, with the height being the computed oscillator strengths. Energy level positions were computed using the SFX2C-1e EOM-CCSD(T)(a)* method, while transition dipole moments were obtained from EOM-CCSD calculations. 
From synchrotrons for XFELs: the soft x-ray near-edge spectrum of the ESCA molecule — Supplementary Material4

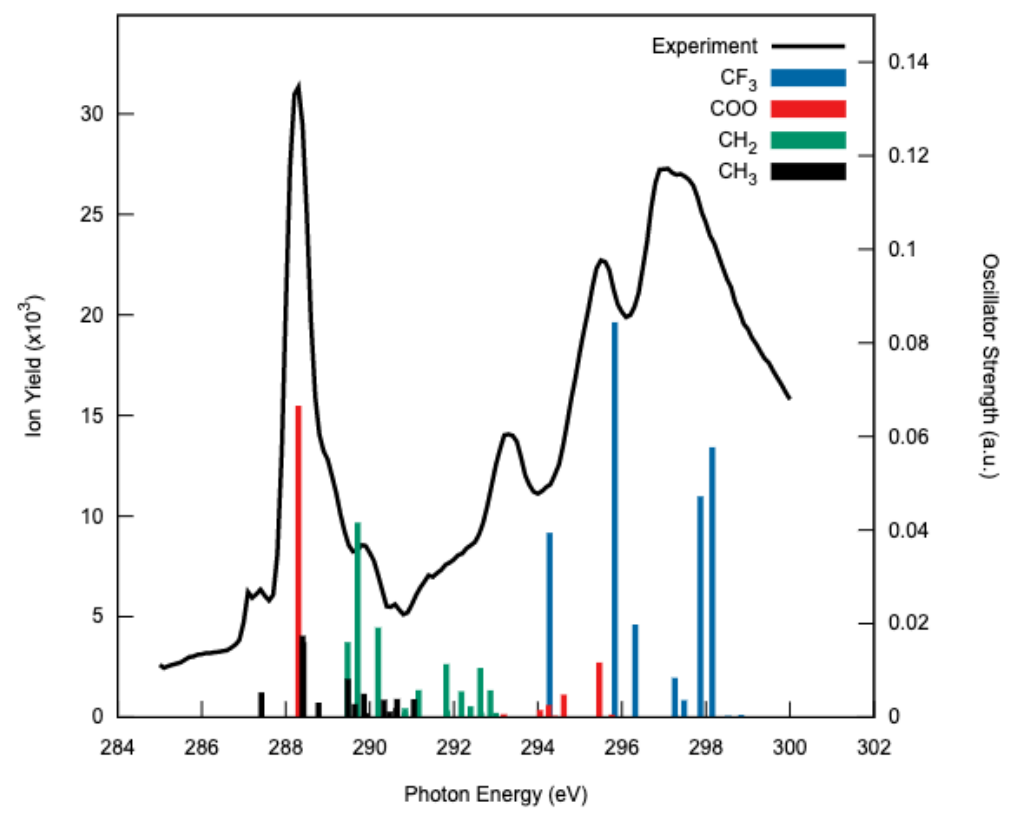

Figure 2: Experimental and computed NEXAFS spectra for the carbon edges of the ethyl trifluoroacetate molecule. The black solid line shows the variation of measured ion yield with respect to photon energies. Blue, red, green, and black sticks represent computed absorption lines for the $\mathrm{CF}_{3}, \mathrm{COO}, \mathrm{CH}_{2}$, and $\mathrm{CH}_{3}$ carbon edges, respectively, with the height being the computed oscillator strengths. Energy levels and transition dipole moments were obtained from EOM-CCSD calculations. The computed spectrum has been red-shifted by $0.81 \mathrm{eV}$ to align the computed $1 \mathrm{~s} \rightarrow \pi^{*}$ transition with the experiment. The cc-pVTZ basis set augmented with two sets of additional diffuse s-, p-, and d-type functions for the targeted carbon and cc-pVTZ basis set for the other atoms have been used in all calculation. 
From synchrotrons for XFELs: the soft x-ray near-edge spectrum of the ESCA molecule — Supplementary Material5

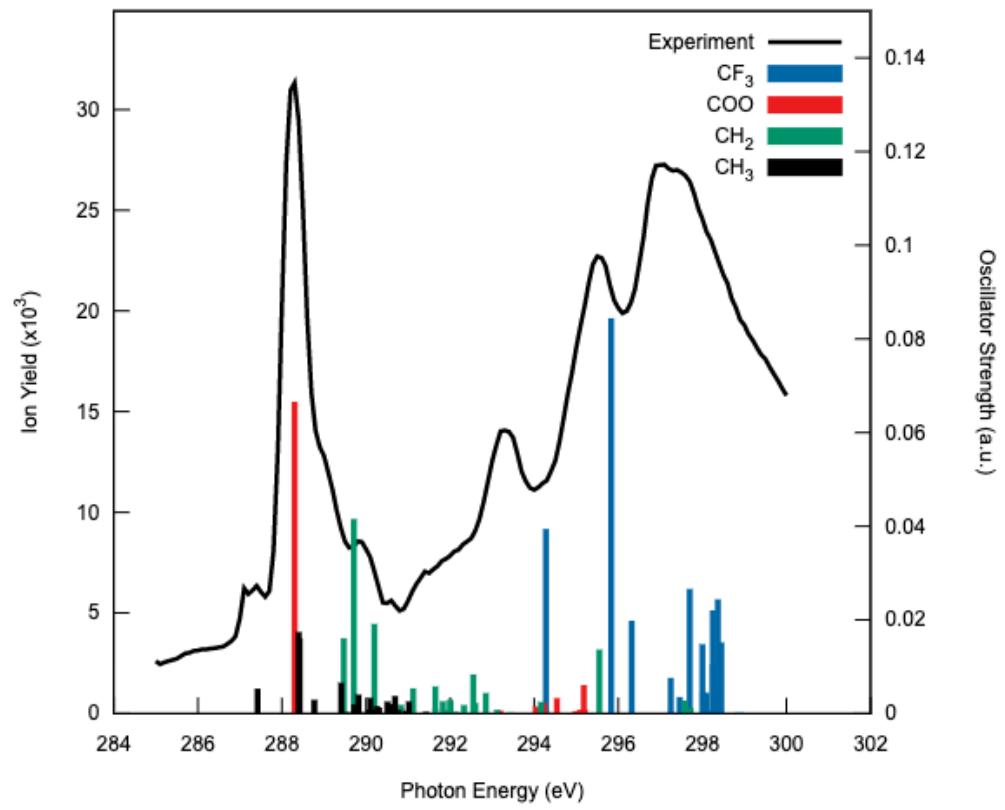

Figure 3: Experimental and computed NEXAFS spectra for the carbon edges of the ethyl trifluoroacetate molecule. The black solid line shows the variation of measured ion yield with respect to photon energies. Blue, red, green, and black sticks represent computed absorption lines for the $\mathrm{CF}_{3}, \mathrm{COO}, \mathrm{CH}_{2}$, and $\mathrm{CH}_{3}$ carbon edges, respectively, with the height being the computed oscillator strengths. Energy levels and transition dipole moments were obtained from EOM-CCSD calculations. The computed spectrum has been red-shifted by $0.81 \mathrm{eV}$ to align the computed $1 \mathrm{~s} \rightarrow \pi^{*}$ transition with the experiment. The cc-pVTZ basis set augmented with three sets of additional diffuse s-, p-, and d-type functions for the targeted carbon and cc-pVTZ basis set for the other atoms have been used in all calculation. 
From synchrotrons for XFELs: the soft x-ray near-edge spectrum of the ESCA molecule — Supplementary Material6

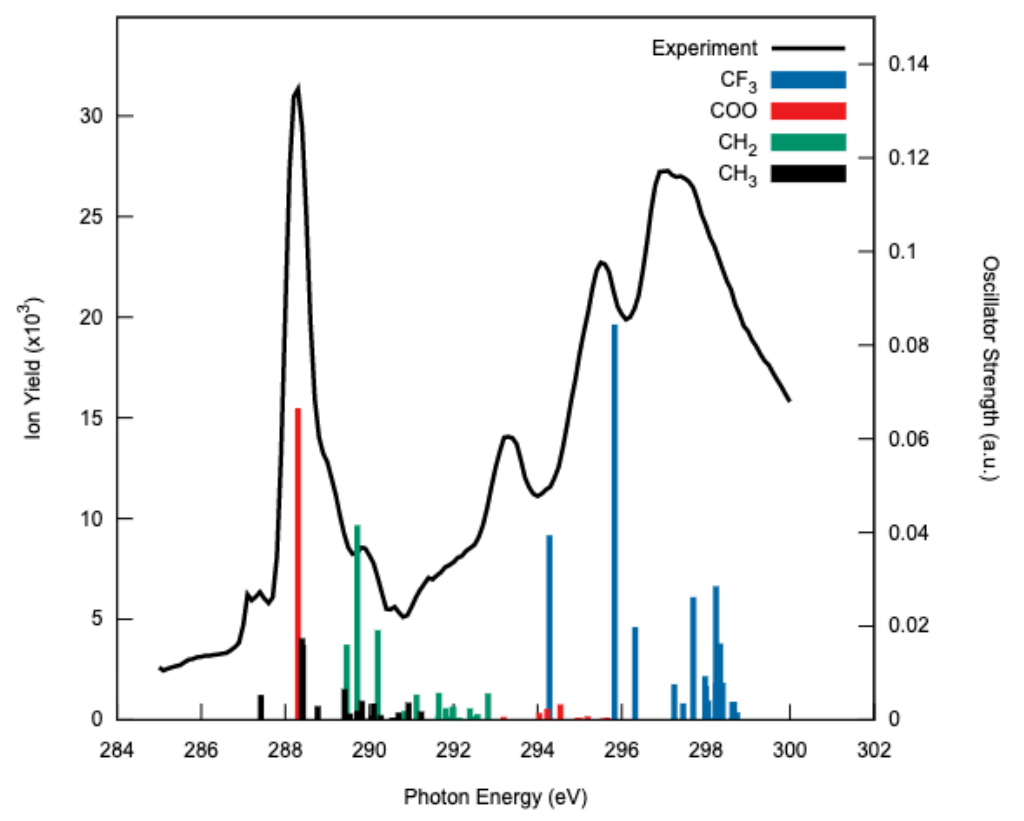

Figure 4: Experimental and computed NEXAFS spectra for the carbon edges of the ethyl trifluoroacetate molecule. The black solid line shows the variation of measured ion yield with respect to photon energies. Blue, red, green, and black sticks represent computed absorption lines for the $\mathrm{CF}_{3}, \mathrm{COO}, \mathrm{CH}_{2}$, and $\mathrm{CH}_{3}$ carbon edges, respectively, with the height being the computed oscillator strengths. Energy levels and transition dipole moments were obtained from EOM-CCSD calculations. The computed spectrum has been red-shifted by $0.81 \mathrm{eV}$ to align the computed $1 \mathrm{~s} \rightarrow \pi^{*}$ transition with the experiment. The cc-pVTZ basis set augmented with four sets of additional diffuse s-, p-, and d-type functions for the targeted carbon and cc-pVTZ basis set for the other atoms have been used in all calculation. 
From synchrotrons for XFELs: the soft x-ray near-edge spectrum of the ESCA molecule — Supplementary Material7

Table 1: Computed transition energies (in $\mathrm{eV}$ ) and oscillator strength (in a.u.) for $\mathrm{CF}_{3}, \mathrm{COO}, \mathrm{CH}_{2}$ and $\mathrm{CH}_{3} \mathrm{C}$ edges of the ESCA molecule used in Figure 3. Energy level positions were computed using the $\operatorname{EOM}-\operatorname{CCSD}(\mathrm{T})(\mathrm{a})^{*}$ method, while transition dipole moments were obtained from EOM-CCSD calculations. Transitions with the higher intensities were specified. The cc-pVTZ basis set augmented with four sets of additional diffuse s-, p-, and d-type functions for the targeted carbon atom and cc-pVTZ basis set for the other atoms have been used in all calculation.

\begin{tabular}{|c|c|c|c|c|}
\hline & \multirow{2}{*}{ Transition } & \multicolumn{2}{|c|}{ Transition energy } & \multirow{2}{*}{ Oscillator strength } \\
\hline & & (original) & (shifted) & \\
\hline \multirow{14}{*}{$\mathrm{CF}_{3}$} & $1 \mathrm{~s} \rightarrow \pi$ & 294.25 & 293.75 & 0.03923 \\
\hline & $1 \mathrm{~s} \rightarrow \mathrm{C}-\mathrm{F} \sigma^{*}$ & 296.10 & 295.60 & 0.08421 \\
\hline & \multirow[t]{3}{*}{$1 \mathrm{~s} \rightarrow 3 p$} & 296.17 & 295.67 & 0.01962 \\
\hline & & 297.01 & 296.51 & 0.00740 \\
\hline & & 297.18 & 296.68 & 0.00332 \\
\hline & \multirow[t]{4}{*}{$1 \mathrm{~s} \rightarrow 3 p$} & 297.53 & 297.03 & 0.02601 \\
\hline & & 297.69 & 297.19 & 0.00913 \\
\hline & & 297.73 & 297.23 & 0.00704 \\
\hline & & 297.80 & 297.30 & 0.00389 \\
\hline & \multirow[t]{5}{*}{$1 \mathrm{~s} \rightarrow 3 p+\pi^{*}$} & 297.99 & 297.49 & 0.02834 \\
\hline & & 298.00 & 297.50 & 0.00758 \\
\hline & & 298.08 & 297.58 & 0.01609 \\
\hline & & 298.09 & 297.59 & 0.00648 \\
\hline & & 298.39 & 297.89 & 0.00366 \\
\hline $\mathrm{COO}$ & $1 \mathrm{~s} \rightarrow \pi^{*}$ & 288.80 & 288.30 & 0.06639 \\
\hline \multirow[t]{6}{*}{$\mathrm{CH}_{2}$} & $1 \mathrm{~s} \rightarrow \mathrm{C}-\mathrm{H} \sigma^{*}$ & 289.51 & 289.01 & 0.01588 \\
\hline & $1 \mathrm{~s} \rightarrow \mathrm{C}-\mathrm{O} \sigma^{*}$ & 289.96 & 289.46 & 0.04136 \\
\hline & \multirow[t]{4}{*}{$1 \mathrm{~s} \rightarrow 3 p$} & 290.22 & 289.72 & 0.01896 \\
\hline & & 291.01 & 290.51 & 0.00520 \\
\hline & & 291.54 & 291.04 & 0.00557 \\
\hline & & 292.70 & 292.20 & 0.00547 \\
\hline \multirow[t]{5}{*}{$\mathrm{CH}_{3}$} & $1 \mathrm{~s} \rightarrow \mathrm{C}-\mathrm{C} \sigma^{*}$ & 287.52 & 287.02 & 0.00513 \\
\hline & $1 \mathrm{~s} \rightarrow 3 p$ & 288.53 & 288.03 & 0.01721 \\
\hline & \multirow[t]{3}{*}{$1 \mathrm{~s} \rightarrow 3 p$} & 288.53 & 288.03 & 0.01581 \\
\hline & & 289.45 & 288.95 & 0.00638 \\
\hline & & 289.75 & 289.25 & 0.00386 \\
\hline
\end{tabular}


From synchrotrons for XFELs: the soft x-ray near-edge spectrum of the ESCA molecule — Supplementary Material8

Table 2: EOM-CCSD values for transition energies (in $\mathrm{eV}$ ) for the nitrogen edge of ammonia used in Figure 2. Oscillator strengths (in a.u.) are enclosed in the parentheses. The transition energies have been red-shifted by $1.3 \mathrm{eV}$ in last column.

\begin{tabular}{|c|c|c|c|c|c|c|}
\hline \multirow{2}{*}{ Transition } & \multirow{2}{*}{ cc-pVTZ } & \multicolumn{5}{|c|}{ cc-pVTZ } \\
\hline & & + spd & $2 \mathrm{spd}$ & $+3 \mathrm{spd}$ & $+4 \mathrm{spd}$ & $+4 \operatorname{spd}($ shifted $)$ \\
\hline \multirow[t]{2}{*}{$1 \mathrm{~s} \rightarrow \sigma^{*}$} & 402.20 & 402.02 & 401.85 & 401.84 & 401.84 & 400.54 \\
\hline & $(0.00680)$ & $(0.00672)$ & $(0.00564)$ & $(0.00565)$ & $(0.00565)$ & $(0.00565)$ \\
\hline \multirow[t]{2}{*}{$1 \mathrm{~s} \rightarrow 3 p(e)$} & 404.07 & 403.97 & 403.51 & 403.50 & 403.50 & 402.20 \\
\hline & $(0.03525)$ & $(0.02908)$ & $(0.01916)$ & $(0.01902)$ & $(0.01903)$ & $(0.01903)$ \\
\hline \multirow[t]{2}{*}{$1 \mathrm{~s} \rightarrow 3 p\left(a_{1}\right)$} & / & 405.03 & 404.15 & 404.13 & 404.13 & 402.83 \\
\hline & & $(0.01267)$ & $(0.00640)$ & $(0.00608)$ & $(0.00608)$ & $(0.00608)$ \\
\hline \multirow[t]{2}{*}{$1 \mathrm{~s} \rightarrow 4 p(e)$} & / & 405.72 & 405.04 & 404.83 & 404.82 & 403.52 \\
\hline & & $(0.00676)$ & $(0.00762)$ & $(0.00382)$ & $(0.00372)$ & $(0.00372)$ \\
\hline \multirow[t]{2}{*}{$1 \mathrm{~s} \rightarrow 4 p\left(a_{1}\right)$} & / & / & 406.09 & 405.54 & 405.49 & 404.19 \\
\hline & & & $(0.00112)$ & $(0.00212)$ & $(0.00172)$ & $(0.00172)$ \\
\hline \multirow[t]{2}{*}{$1 \mathrm{~s} \rightarrow 5 p(e)$} & / & / & 405.98 & 405.40 & 405.34 & 404.04 \\
\hline & & & $(0.00121)$ & $(0.00222)$ & $(0.00273)$ & $(0.00273)$ \\
\hline \multirow[t]{2}{*}{$1 \mathrm{~s} \rightarrow 6 p(e)$} & / & / & 406.68 & 406.07 & 405.70 & 404.40 \\
\hline & & & $(0.00560)$ & $(0.00704)$ & $(0.00111)$ & (0.00111) \\
\hline
\end{tabular}


From synchrotrons for XFELs: the soft x-ray near-edge spectrum of the ESCA molecule — Supplementary Material9

Table 3: EOM-CCSD values for transition energies (in $\mathrm{eV}$ ) and oscillator strengths for the $\mathrm{C}_{s}$ and $\mathrm{C}_{1}$ conformers for the pre-methyl carbon edge. Note that the separation between $\mathrm{COO}$ $1 s \rightarrow \pi^{*}$ and $\mathrm{CH}_{2} 1 s \rightarrow \sigma^{*}$ transitions (the first and third lines) is $1.41 \mathrm{eV}$ for the $\mathrm{C}_{s}$ conformer and $1.37 \mathrm{eV}$ for the $\mathrm{C}_{1}$ conformer. The ccpVTZ basis set augmented with two sets of additional diffuse s-, p-, and d-type functions for the targeted carbon atom and cc-pVTZ basis set for the other atoms have been used in all calculation.

\begin{tabular}{cccc}
\hline \multicolumn{2}{c}{ Transition energies } & \multicolumn{2}{c}{ Oscillator } \\
\hline $\mathrm{C}_{s}$ & $\mathrm{C}_{1}$ & $\mathrm{C}_{s}$ & $\mathrm{C}_{1}$ \\
289.11 & 289.12 & 0.66400 & 0.06464 \\
290.28 & 290.28 & 0.01254 & 0.01734 \\
290.52 & 290.49 & 0.03833 & 0.03376 \\
291.01 & 291.14 & 0.01895 & 0.01983 \\
291.64 & 291.44 & 0.00090 & 0.00096 \\
288.23 & 288.24 & 0.00391 & 0.00404 \\
289.21 & 289.15 & 0.01219 & 0.01014 \\
289.23 & 289.31 & 0.01579 & 0.01011 \\
289.59 & 289.69 & 0.00235 & 0.00518 \\
$/$ & 290.29 & $/$ & 0.00437 \\
290.29 & 290.33 & 0.00796 & 0.00882 \\
290.45 & 290.5 & 0.00262 & 0.00075 \\
290.67 & 290.63 & 0.00385 & 0.00211 \\
$/$ & 290.82 & $/$ & 0.00218 \\
\hline
\end{tabular}

